Department of Social Systems and Management

\author{
Discussion Paper Series
}

No. 1241

Constant Rebalanced Portfolio Optimization under Nonlinear Transaction Costs

by

Yuichi Takano and Jun-ya Gotoh

July 2009

UNIVERSITY OF TSUKUBA

Tsukuba, Ibaraki 305-8573

JAPAN 


\title{
Constant Rebalanced Portfolio Optimization under Nonlinear Transaction Costs
}

\author{
Yuichi Takano *† Jun-ya Gotoh ${ }^{\ddagger}$
}

July 15, 2009

\begin{abstract}
In this paper, we study a multi-period portfolio optimization where conditional value-atrisk $(\mathrm{CVaR})$ is controlled as well as expected return, and the so-called constant rebalancing strategy is employed under nonlinear transaction costs. In general, the optimization of this strategy itself is, however, difficult to attain a globally optimal solution because of the nonconvexity. In addition, nonlinearity of the transaction cost and CVaR functions makes things worse, and even a locally optimal solution may not be reached via a state-of-the-art nonlinear programming solver when the size of the problem is large. In order to provide a practical solution to the highly complex problem, we propose a local search algorithm where linear approximation problems and nonlinear equations are iteratively solved. Computational results are presented, showing that the proposed algorithm attains a good solution in practical time even when a revised version of an existing global optimization approach cannot return any feasible solutions.
\end{abstract}

Keywords: Multi-period portfolio optimization, Constant rebalancing, Transaction cost, Conditional value-at-risk, Market impact cost

\section{Introduction}

Multi-Period Portfolio Optimization. Since the seminal work of Markowitz (1952), optimization methods for portfolio selection have been actively studied and playing an important role in financial decision makings (see, e.g., Cornuejols and Tütüncü, 2007). Since the early stage, it has been recognized that multi-period model is essential for long-term portfolio management (e.g., Markowitz, 1991) as well as the single-period models as in Markowitz (1952). It was, however, difficult at that time to solve even single-period problems because of the computational

\footnotetext{
${ }^{*}$ Graduate School of Systems and Information Engineering, University of Tsukuba, 1-1-1 Tennoudai, Tsukuba, Ibaraki 305-8573, Japan. e-mail: takano10@sk.tsukuba.ac.jp

${ }^{\dagger}$ Research Fellow of the Japan Society for the Promotion of Science

${ }^{\ddagger}$ Department of Industrial and Systems Engineering, Chuo University, 1-13-27 Kasuga, Bunkyo-ku, Tokyo 112-8551, Japan. e-mail: jgoto@indsys.chuo-u.ac.jp
} 
burden, and accordingly, recent developments of computational environment and stochastic programming models had been required for constructing a multi-period portfolio. Intractability of multi-period models depends on how to describe uncertainty in values of investable assets and on how to formulate investment strategies.

Description of Uncertainty for Multi-Period Model. Multi-period portfolio optimization was first introduced as a dynamic stochastic programming (stochastic control problem) (e.g., Merton, 1969; Samuelson, 1969; Merton, 1971) (see Infanger, 2006, for detailed references). Although closed-form solutions to those problems can be derived under strong assumptions, such an approach cannot be easily generalized in the presence of market frictions, e.g., transaction costs. Moreover, it requires to overcome a heavy computational burden for actual numerical implementation (Brennan et al., 1997; Brandt, 1999), and therefore, alternative various stochastic programming models have been proposed for multi-period portfolio optimization (e.g., Kusy and Ziemba, 1986; Mulvey and Vladimirou, 1989; Dantzig and Infanger, 1993; Cariño et al., 1994). Mainly studied models, e.g., in Mulvey and Ziemba (1995); Ziemba and Mulvey (1998), employs a scenario tree for representing the uncertainty of asset values. However, seeking accuracy in the uncertainty representation, the size of the optimization problem may grow exponentially (Ermoliev and Wets, 1988). On the other hand, simulated path model, in which scenarios are represented by sample paths generated by a Monte Carlo simulation method, yields better accuracy in describing uncertainty (Hibiki, 2003). Incorporating the benefits of the two approaches, Hibiki (2003) proposed a hybrid (bundling simulated path) model which is designed not only to describe uncertainty on a simulated path structure but also to enable one to make conditional decisions in a tree structure. In this paper, taking into account the compatibility with constant rebalancing strategy, the simulated path model is employed.

Advantages of Constant Rebalancing Strategy. Among investment strategies for longterm asset management, buy-and-hold and periodical rebalancing are two of the most popular policies (see Perold and Sharpe, 1995, for detailed discussion on various investment strategies). The former is an investment strategy in which an investor buys financial assets and holds them throughout the planning period. On the other hand, rebalancing strategy is a dynamic approach to investment decision making, and the most familiar one is constant rebalancing (in other words, fixed mix, constant mix and the like). This rebalancing strategy requires purchase and sale of 
assets so that investment proportion would be restored to the original one at the beginning of each period.

The advantages of constant rebalancing are as follows:

1. [Advantage as an Optimization Model.] In formulating the rebalancing on a simulated path model, we can do without introducing decision variables of investment proportion separately for each period, which means that only variables of the number of assets are required.

2. [Advantage for Financial Institutions.] In practice, it is easy to explain this rebalancing policy to customers due to its simplicity.

3. [Validity as an Investment Strategy.] The constant rebalancing is a kind of contrarian investment strategy, which suggests to purchase assets whose price has decreased and to sell ones whose price has increased, and it is widely believed among investors that it is effective for mid/long term investment.

Solution to Constant Rebalanced Portfolio Optimization. Multi-period portfolio optimization with constant rebalancing strategy is relatively easy to solve in case of log-optimal portfolio (Cover, 1991), in which the asymptotically optimal portfolio is determined by maximizing the expected log return. However, it becomes a nonconvex problem and difficult to attain a globally optimal solution in general when a risk measure (e.g., variance of returns) is introduced (Maranas et al., 1997). Moreover, the problems with various constraints and objectives are much harder to solve. In addition, if the problem size is large, any locally optimal solutions may not be reached via state-of-the-art nonlinear programming (NLP) solvers. Maranas et al. (1997) considered multi-period mean-variance portfolio optimization with constant rebalancing strategy for long-term financial planning. They proposed a rectangular branch-and-bound algorithm in order to globally solve this problem. By enjoying the fact that the number of assets is only up to nine, their deterministic algorithm attains a globally optimal solution in practical time. However, transaction costs are not considered in Maranas et al. (1997) and cannot be easily dealt with in their framework because introducing cost functions would prevent the problem from having a compact representation they enjoyed. Hibiki (2006) proposed an iterative optimization algorithm by alternately fixing decision variables for approximately solving the hybrid model with a fixed-proportion strategy. In this strategy, investment proportions have the same value for all simulated paths passing the same bundle of states. Although this algorithm works 
well by employing a good initial solution, it may not work in the constant rebalanced portfolio optimization in question because initial solution can be hardly improved due to the excessive reduction of degree of freedom. Besides, transaction costs are not considered in Hibiki (2006).

Transaction Costs. Transaction costs have also been a subject of concerns in the literature, and should be taken into consideration for successful investing in practice. In particular, a large amount of transactions usually make asset price move in an unfavorable direction. Such an effect is known as market impact, and the associated cost is nonnegligible for institutional investors. In this paper, we consider a convex cost function which is supposed to represent market impact. Among recent papers on multi-period portfolio optimization under transaction costs are the model minimizing one-sided deviation measure (Pinar, 2007), the robust optimization approach (Bertsimas and Pachamanova, 2008), the policy optimization approach (Calafiore, 2008; Skaf and Boyd, 2008), and the like. However in those papers, constant rebalancing strategy is not considered, and only linear transaction costs are considered. Gaivoronski and Stella (2003) proposed a log-optimal portfolio with transaction costs for an adaptive portfolio selection policy. Zhang and Zhang (2009) proposed a hybrid model under linear transaction costs in which CVaR is employed as a risk measure, and solve the resulting nonconvex program by applying a genetic algorithm.

The purpose of this paper is to propose a local search algorithm for solving the constant rebalanced portfolio optimization under nonlinear transaction costs. In this algorithm, linear approximation problems and nonlinear equations are iteratively solved via linear programming (LP) solver and Newton's method respectively. In contrast to the use of nonlinear programming solver, the proposed strategy can provide a solution to the complex problem. Moreover, an incumbent solution can be improved better than the alternating optimization (Hibiki, 2006). The effectiveness of the proposed local search algorithm is examined through computational experiments where the performance is compared to the buy-and-hold strategy.

The rest of the paper is organized as follows. In Section 2, a mathematical description of a constant rebalancing model under transaction costs is given. Section 3 explains the portfolio optimization problems via conditional value-at-risk (CVaR) minimization, and in Section 4, the local search algorithm for solving them is proposed. Computational results are presented in Section 5, showing the comparative superiority of the proposed approach and the constant 


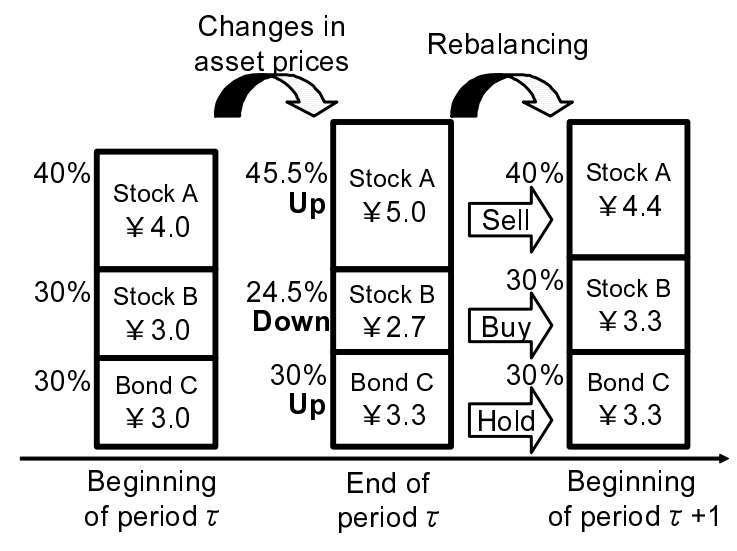

Figure 1: Example of Constant Rebalancing

rebalancing strategy.

\section{Constant Rebalancing under Transaction Costs}

In this section, we give a mathematical description of a constant rebalancing model under transaction costs.

To begin with, let us define the index sets as follows:

$I:=\{1, \ldots, n\} \quad: \quad$ index set of investable financial assets

$T:=\{1, \ldots, t\} \quad: \quad$ index set of time periods in the future

$J:=\{1, \ldots, m\} \quad$ : index set of given scenarios, or in other words, index set of simulated paths (see Figure 2).

Let $\gamma_{i}: \mathbb{R} \rightarrow \mathbb{R}$ be a function representing the transaction cost of asset $i \in I$ for the amount of transactions $\xi$. In this paper, we assume that $\gamma_{i}$ is a convex function representing a market impact cost:

$$
\gamma_{i}(\xi):=a_{i}[-\xi]^{+} \exp \left(-b_{i} \xi\right)+c_{i}[\xi]^{+} \exp \left(d_{i} \xi\right)
$$

where $a_{i}, b_{i}, c_{i}, d_{i}(i \in I)$ are non-negative parameters to be estimated, and $[\xi]^{+}:=\max \{\xi, 0\}$. The value of $\gamma_{i}$ is almost zero in case of small amount of transactions and grows exponentially as a function of the amount of transactions. Besides, the linear transaction costs which are considered in, e.g., Pinar (2007); Bertsimas and Pachamanova (2008); Calafiore (2008); Skaf and Boyd (2008) can be represented by placing zero as $b_{i}$ and $d_{i}$. 
Figure 1 shows an example of constant rebalancing. Let us assume that 4.0, 3.0 and 3.0 billion Japanese yen are invested in Stock A, Stock B and Bond C respectively at the beginning of a period. In a period, investment proportion will be different from the initial one, i.e., $40 \%, 30 \%$ and $30 \%$ due to the changes in asset prices. Then, the constant rebalancing strategy compels to purchase assets whose price has decreased and to sell assets whose price has increased so that the proportion is restored to be $40 \%, 30 \%$ and $30 \%$ at the beginning of the next period.

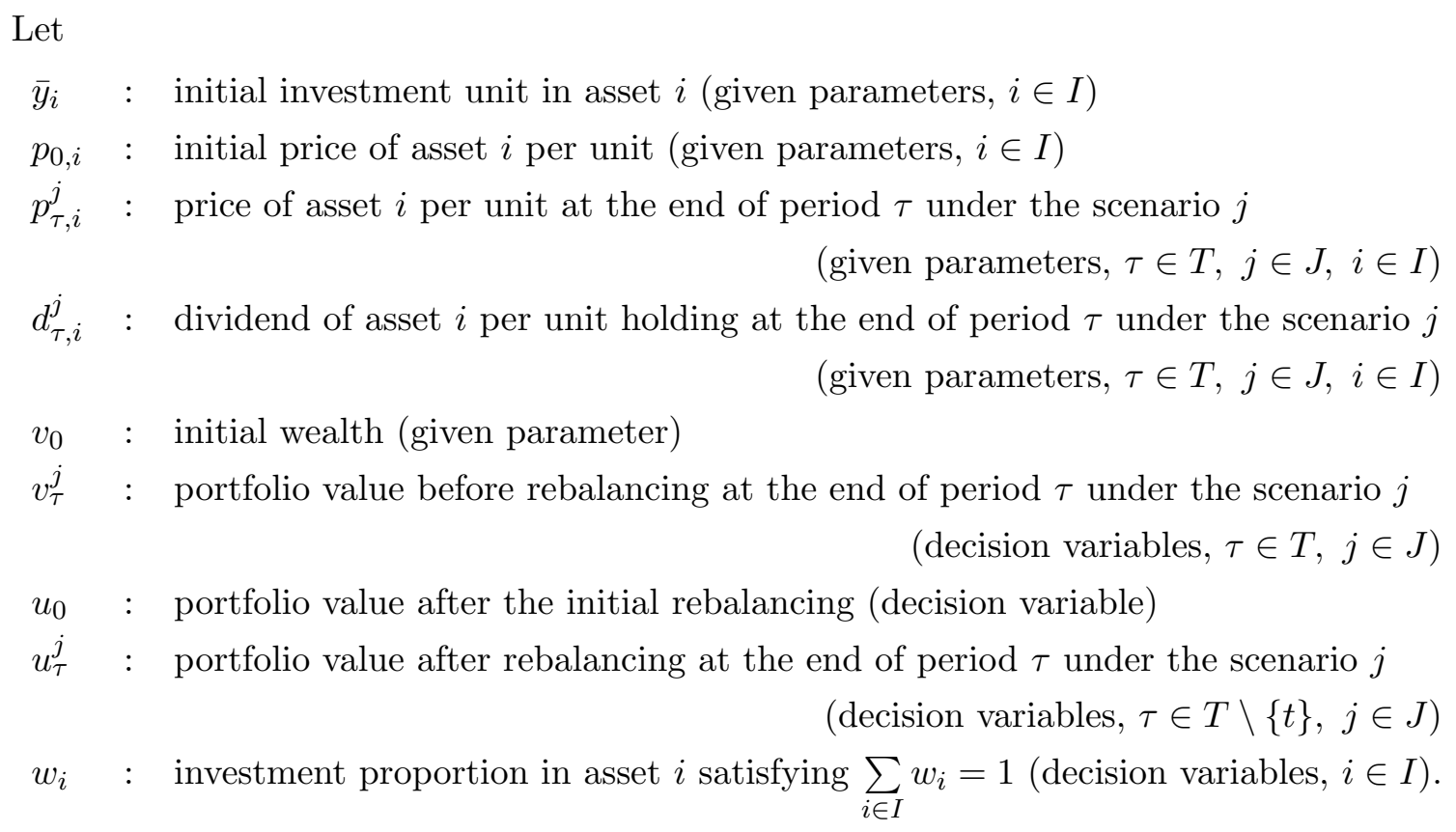

We assume that the investor has an initial portfolio $\overline{\boldsymbol{y}}$ at the beginning of the investment. The constant rebalancing strategy enforces the rebalancing to a constant proportion $\boldsymbol{w}$ at the beginning of each discrete investment period. In case of initial rebalancing, the invested amount $p_{0, i} \bar{y}_{i}$ is adjusted to $u_{0} w_{i}$, and at the same time, portfolio value $u_{0}$ is calculated by subtracting the transaction cost from initial wealth $v_{0}$. The relation between initial wealth $v_{0}$ and portfolio value $u_{0}$ is given by

$$
v_{0}=u_{0}+\sum_{i \in I} \gamma_{i}\left(u_{0} w_{i}-p_{0, i} \bar{y}_{i}\right)
$$

Due to asset price changes and receipt of dividends, the portfolio value changes over the period 1. Accordingly, portfolio value before rebalancing at the end of period 1 under the scenario $j$ is given by

$$
v_{1}^{j}=u_{0} \sum_{i \in I}\left(1+x_{1, i}^{j}\right) w_{i}
$$




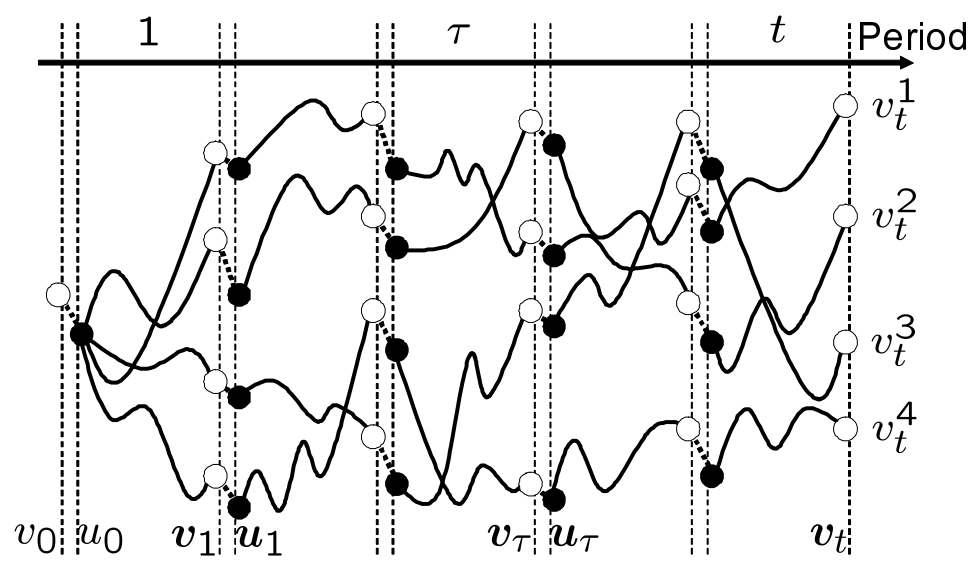

Figure 2: Simulated Paths of Portfolio Value

where $x_{\tau, i}^{j}$ is calculated as follows:

$$
x_{1, i}^{j}:=\frac{p_{1, i}^{j}-p_{0, i}+d_{1, i}^{j}}{p_{0, i}} ; \quad x_{\tau, i}^{j}:=\frac{p_{\tau, i}^{j}-p_{\tau-1, i}^{j}+d_{\tau, i}^{j}}{p_{\tau-1, i}^{j}}, \quad \tau \in T \backslash\{1\},
$$

and represents the return of asset $i$ at the period $\tau$ under the scenario $j$.

Taking it into consideration that the investment unit in asset $i$ right before the rebalancing is $\left(u_{0} w_{i}\right) / p_{0, i}$, in the same way as Equation (2), the relation between portfolio values $v_{1}^{j}$ and $u_{1}^{j}$ under the scenario $j$ is given by

$$
v_{1}^{j}=u_{1}^{j}+\sum_{i \in I} \gamma_{i}\left(u_{1}^{j} w_{i}-\frac{p_{1, i}^{j} u_{0} w_{i}}{p_{0, i}}\right) .
$$

Similarly, portfolio value before rebalancing at the end of period $\tau \in T \backslash\{1\}$ under the scenario $j$ is given by

$$
v_{\tau}^{j}=u_{\tau-1}^{j} \sum_{i \in I}\left(1+x_{\tau, i}^{j}\right) w_{i}
$$

and the relation between portfolio values $v_{\tau}^{j}$ and $u_{\tau}^{j}$ at the end of period $\tau \in T \backslash\{1, t\}$ under the scenario $j$ is given by

$$
v_{\tau}^{j}=u_{\tau}^{j}+\sum_{i \in I} \gamma_{i}\left(u_{\tau}^{j} w_{i}-\frac{p_{\tau, i}^{j} u_{\tau-1}^{j} w_{i}}{p_{\tau-1, i}^{j}}\right) .
$$

In Figure 2, an example of changes in portfolio value is illustrated. The portfolio value falls at the beginning of each period due to transaction costs associated with the rebalancing (see Equations (2), (5) and (7)). 


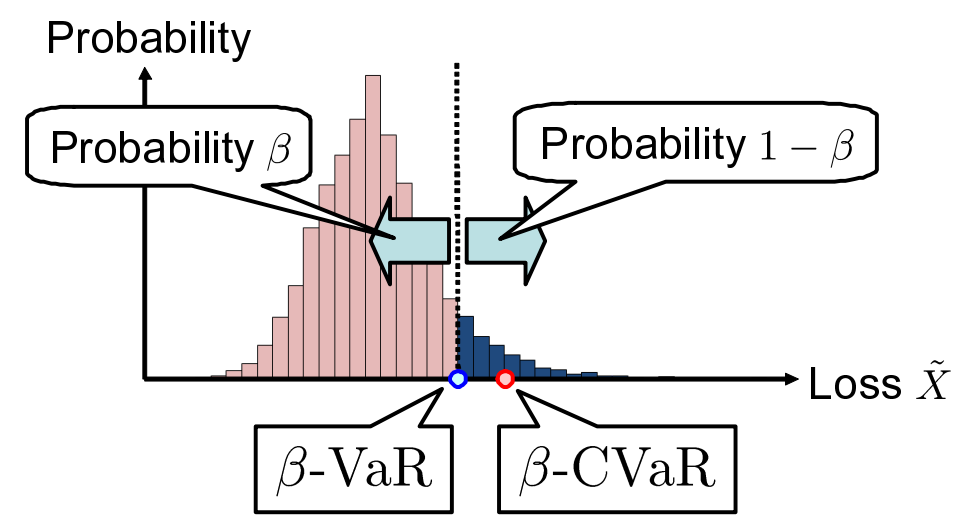

Figure 3: Value-at Risk and Conditional Value-at-Risk

\section{Conditional Value-at-Risk and Portfolio Optimization Prob- lem}

In this section, we introduce a risk measure known as conditional value-at-risk (CVaR), and formulate a constant rebalanced portfolio optimization problem in which CVaR is controlled as well as expected return.

\subsection{Conditional Value-at-Risk}

Portfolio optimization problem is usually formulated via two-parameter approach as in the Markowitz's mean-variance model (Markowitz, 1952) where the two parameters represent expected return and variance of a portfolio return. Among various risk measures examined in the literature (see, e.g., Cornuejols and Tütüncü, 2007), we employ conditional value-at-risk $(\mathrm{CVaR})$. Let $\beta \in(0,1)$ be a parameter representing confidence level. (In numerical experiments of Section 5 , we set $\beta=0.95$.) $\beta$-CVaR is then explained as the conditional expectation of random loss $\tilde{X}$ exceeding value-at-risk (VaR), the $\beta$-quantile of the loss, as Figure 3 indicates. It is worth noting that $\mathrm{CVaR}$ has various desirable properties as a risk measure in computational and theoretical aspects (see, e.g., Pflug, 2000; Rockafellar and Uryasev, 2002).

If the support or realizations of random loss $\tilde{X}$ is given by a finite set $\left\{X_{j} \mid j \in J\right\}, \beta$-CVaR is evaluated by the optimal value of the following convex program (Rockafellar and Uryasev, 2002):

$$
\mid \underset{\rho \in \mathbb{R}}{\operatorname{minimize}} \quad \rho+\frac{1}{1-\beta} \sum_{j \in J} p_{j}\left[X_{j}-\rho\right]^{+},
$$


where $p_{j}$ are the occurrence probability of scenario $j \in J$. Moreover, Problem (8) can be rewritten as the following linear programming problem (Rockafellar and Uryasev, 2002):

$$
\mid \begin{array}{ll}
\underset{(\rho, \boldsymbol{z}) \in \mathbb{R} \times \mathbb{R}^{m}}{\operatorname{minimize}} & \rho+\frac{1}{1-\beta} \sum_{j \in J} p_{j} z_{j} \\
\text { subject to } & z_{j} \geq X_{j}-\rho, \quad z_{j} \geq 0, \quad j \in J .
\end{array}
$$

\subsection{Formulation}

In the rest part of the paper, we assume that occurrence probability of scenario $j \in J=\{1, \ldots, m\}$ is $1 / m$. In the formulation, we consider the following constraints:

\section{Portfolio Dynamics Equation}

$$
\left\{\begin{array}{lrl}
v_{0}=u_{0}+\sum_{i \in I} \gamma_{i}\left(u_{0} w_{i}-p_{0, i} \bar{y}_{i}\right), & \\
v_{1}^{j}=u_{1}^{j}+\sum_{i \in I} \gamma_{i}\left(u_{1}^{j} w_{i}-\frac{p_{1, i}^{j} u_{0} w_{i}}{p_{0, i}}\right), & & \cdots(10 . \mathrm{a}) \\
v_{\tau}^{j}=u_{\tau}^{j}+\sum_{i \in I} \gamma_{i}\left(u_{\tau}^{j} w_{i}-\frac{p_{\tau, i}^{j} u_{\tau-1}^{j} w_{i}}{p_{\tau-1, i}^{j}}\right), & & \cdots(10 . \mathrm{b}) \\
v_{1}^{j}=u_{0} \sum_{i \in I}\left(1+x_{1, i}^{j}\right) w_{i}, & j \backslash\{1, t\}, j \in J & \cdots(10 . \mathrm{c}) \\
v_{\tau}^{j}=u_{\tau-1}^{j} \sum_{i \in I}\left(1+x_{\tau, i}^{j}\right) w_{i}, & \tau \in T \backslash\{1\}, j \in J & \cdots(10 . \mathrm{d})
\end{array}\right.
$$

\section{Investment Proportion Constraint}

$$
\begin{cases}w_{i}^{L} \leq w_{i} \leq w_{i}^{U}, \quad i \in I & \cdots(11 . \mathrm{a}) \\ \sum_{i \in I} w_{i}=1, & \cdots(11 . \mathrm{b})\end{cases}
$$

where $w_{i}^{L}\left(w_{i}^{U}\right)$ is a lower (upper) limit of investment proportion in asset $i \in I$.

In the following formulation, the random loss regarding the definition of CVaR is defined as " $(-1) \times($ portfolio value at the end of period $t)$," that is, $-v_{t}^{j}$, and both the maximization of the expected portfolio value at the end of period $t$ and the minimization of CVaR are considered at the same time via an objective function:

$$
\operatorname{maximize}_{\boldsymbol{u}, \boldsymbol{v}, \rho, \boldsymbol{w}} \lambda\left(\frac{1}{m} \sum_{j \in J} v_{t}^{j}\right)-(1-\lambda)\left(\rho+\frac{1}{(1-\beta) m} \sum_{j \in J}\left[-v_{t}^{j}-\rho\right]^{+}\right)
$$

subject to Portfolio Dynamics Equation (10),

Investment Proportion Constraint (11), 


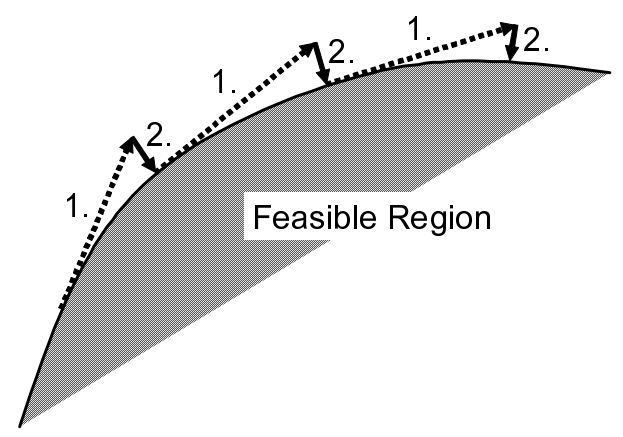

1. Solving a linear approximation problem, 2. Solving nonlinear equations

Figure 4: Geometric Interpretation of the Local Search Algorithm

where $\lambda \in[0,1]$ is a trade-off parameter between the expected return and the CVaR.

A large number of bilinear terms of decision variables $\left(u_{0} w_{i}, u_{\tau}^{j} w_{i}\right.$ and the like) appear in Constraint (10). Therefore, Problem (12) is nonconvex and difficult to attain a globally optimal solution in general (Konno et al., 1997; Tuy, 1998). Moreover, in particular if the number of scenarios is so large, the problem size becomes huge, and consequently, even a state-of-the-art NLP solver such as NUOPT may not provide any solutions. Thus in this paper, we propose an iterative local search algorithm repeating the following two steps (Figure 4):

1. Solving a linear approximation problem for problem (12)

2. Solving nonlinear equations (10) via Newton's method

Fleten et al. (2002) stated that for their data sets, constant rebalanced portfolio optimization problems are virtually convex since their local search method using many different starting values for each instance always converged to the same solution. Although in their problem, risk is measured by the expected accumulated quadratic shortfalls and transaction costs are not considered, their statement on the convexity of the problem motivates us to apply a local search approach to Problem (12). Since we only solve an "approximation" problem for Problem (12), the obtained solution does not necessarily satisfy Constraint (10). Therefore, we need to find a feasible solution via Newton's method where a solution to the approximation problem is employed as a starting value. 
In addition, our local search algorithm is suited to more general formulations. For example, let us consider the following problem

$$
\begin{array}{|ll}
\underset{\boldsymbol{u}, \boldsymbol{v}, \rho, \boldsymbol{w}}{\operatorname{maximize}} & \frac{1}{m} \sum_{j \in J} v_{t}^{j} \\
\text { subject to } & \rho_{1}+\frac{1}{(1-\beta) m} \sum_{j \in J}\left[v_{0}-v_{1}^{j}-\rho_{1}\right]^{+} \leq \alpha_{1}, \\
& \rho_{\tau}+\frac{1}{(1-\beta) m} \sum_{j \in J}\left[v_{\tau-1}^{j}-v_{\tau}^{j}-\rho_{\tau}\right]^{+} \leq \alpha_{\tau}, \quad \tau \in T \backslash\{1\} \\
& \text { Portfolio Dynamics Equation (10), } \\
& \text { Investment Proportion Constraint (11), }
\end{array}
$$

where $\alpha_{\tau}$ are user-defined parameters for representing upper bounds of CVaR value at the end of each period $\tau \in T$, and $\beta$-CVaR at each period is defined in terms of the "decrease in portfolio value during the period $\tau$," that is, $v_{\tau-1}^{j}-v_{\tau}^{j}$. Imposing CVaR constraints on each rebalancing is meaningful in practice since it is frequently uncertain when long-term investment will be discontinued due to a large unrealized loss.

\subsection{Other Algorithms in the Literature}

Maranas et al. (1997) proposed a rectangular branch-and-bound algorithm for the constant rebalanced mean-variance portfolio optimization under no transaction costs by enjoying the fact that the constraints for representing the portfolio dynamics can be moved to the objective by eliminating the associated decision variables (see Section 5.1.1 for detailed explanation). However, such a variable elimination is impossible under transaction costs, and therefore their algorithm cannot be applied to Problem (12). In addition, even if there are no transaction costs, the performance of the branch-and-bound procedure for Problem (13) deteriorates since the size of subproblems becomes large due to the CVaR constraints.

On the other hand, a typical heuristic algorithm for the problem, in which a large number of bilinear terms of decision variables appear, is an iterative optimization by alternately fixing decision variables, $\boldsymbol{w}$ and $\boldsymbol{u}$, which compose the bilinear terms. For instance, given $\overline{\boldsymbol{w}}$, Problem (12) is solved subject to $\boldsymbol{w}=\overline{\boldsymbol{w}}$, which is a convex program. Then, for the obtained solution $\overline{\boldsymbol{u}}$, Problem (12) is solved subject to $\boldsymbol{u}=\overline{\boldsymbol{u}}$, resulting in a new $\overline{\boldsymbol{w}}$. By repeating this procedure, the sequence of the obtained solutions is expected to improve. However, if decision variable $\boldsymbol{u}$ is fixed, degree of freedom of the other variables is excessively reduced as well. As a result, local 
search of Problem (12) subject to $\boldsymbol{u}=\overline{\boldsymbol{u}}$ provides a solution $\boldsymbol{w}$ which is almost the same as in the former iteration, and therefore the sequence of the obtained solutions is not improved enough. Hibiki (2006) proposed an alternating optimization for approximately solving his model. His algorithm works well if a good initial solution is obtained. For the problem in hand, however, this alternating strategy fails to improve the incumbent solutions due to the excessive reduction of the feasibility of the subproblems. In addition, transaction costs are not considered in Hibiki (2006).

\section{Local Search Algorithm}

In this section, we explain the local search algorithm for solving Problem (12) in detail. The algorithm consists of two procedures: (i) solving a linear approximation problem, and (ii) finding a feasible solution.

\subsection{Linear Approximation Problem for Problem (12)}

The linear approximation problem for Problem (12), called LAP $(\overline{\boldsymbol{u}}, \overline{\boldsymbol{w}})$, is formulated as follows. First, the objective function is linearized by introducing auxiliary variables $z_{t}^{j}$ (see the relation between Problems (8) and (9)). Next, the nonlinear terms of decision variables in Constraint (10) are approximated in a linear manner at $(\overline{\boldsymbol{u}}, \overline{\boldsymbol{w}})$ with respect to $\boldsymbol{u}, \boldsymbol{w}$ (see Appendix A). As a result, $\operatorname{LAP}(\overline{\boldsymbol{u}}, \overline{\boldsymbol{w}})$ is formulated as the following problem:

$$
\operatorname{LAP}(\overline{\boldsymbol{u}}, \overline{\boldsymbol{w}}) \mid \begin{array}{ll}
\operatorname{maximize}_{\boldsymbol{u}, \boldsymbol{v}, \rho, \boldsymbol{w}, \boldsymbol{z}} & \lambda\left(\frac{1}{m} \sum_{j \in J} v_{t}^{j}\right)-(1-\lambda)\left(\rho+\frac{1}{(1-\beta) m} \sum_{j \in J} z_{t}^{j}\right) \\
\text { subject to } & z_{t}^{j} \geq-v_{t}^{j}-\rho, \quad z_{t}^{j} \geq 0, \\
& \text { Linearly Approximated Portfolio Dynamics Equation (19), } \\
& \text { Investment Proportion Constraint (11), } \\
& \boldsymbol{w} \in \mathcal{N}(\overline{\boldsymbol{w}}),
\end{array}
$$

where $\mathcal{N}(\overline{\boldsymbol{w}})$ is a neighborhood of $\overline{\boldsymbol{w}}$, given by

$$
\mathcal{N}(\overline{\boldsymbol{w}}):=\left\{\boldsymbol{w} \mid \bar{w}_{i}-\kappa_{i} \leq w_{i} \leq \bar{w}_{i}+\kappa_{i}, i \in I\right\},
$$

where $\kappa_{i}>0$ are step size parameters regarding investment proportion of asset $i \in I$. The constraint $\boldsymbol{w} \in \mathcal{N}(\overline{\boldsymbol{w}})$ is imposed so that the solution to the linearly approximated problem will not be far from the incumbent point $(\overline{\boldsymbol{u}}, \overline{\boldsymbol{w}})$. 


\subsection{Finding a Feasible Solution via Newton's Method}

In order to approximate Problem (12) in a linear manner, we need a feasible $(\overline{\boldsymbol{u}}, \overline{\boldsymbol{w}})$ at each iteration, but a solution to $\operatorname{LAP}(\overline{\boldsymbol{u}}, \overline{\boldsymbol{w}})$ does not necessarily satisfy Constraint (10). In the following, we explain the procedure for finding a feasible $(\overline{\boldsymbol{u}}, \overline{\boldsymbol{w}})$ by starting from a (possibly infeasible) solution of $\operatorname{LAP}(\overline{\boldsymbol{u}}, \overline{\boldsymbol{w}})$.

Let $\left(\boldsymbol{u}^{\mathrm{LAP}}, \boldsymbol{v}^{\mathrm{LAP}}, \rho^{\mathrm{LAP}}, \boldsymbol{w}^{\mathrm{LAP}}, \boldsymbol{z}^{\mathrm{LAP}}\right)$ be a solution to $\operatorname{LAP}(\overline{\boldsymbol{u}}, \overline{\boldsymbol{w}})$. Obviously, $\boldsymbol{w}^{\mathrm{LAP}}$ is feasible to Problem (12) since Constraint (11) is included in $\operatorname{LAP}(\overline{\boldsymbol{u}}, \overline{\boldsymbol{w}})$. In the procedure explained below, we find a feasible solution $\left(\boldsymbol{w}^{\mathrm{LAP}}, \overline{\boldsymbol{u}}, \overline{\boldsymbol{v}}\right)$ to Problem (12) by substituting $\boldsymbol{w}^{\mathrm{LAP}}$ into Constraint (10) and solving the nonlinear equations in $\boldsymbol{u}$ and $\boldsymbol{v}$.

1. Finding feasible $u_{0}$ via Constraint (10.a). In Constraint (10.a), if we substitute $\boldsymbol{w}^{\mathrm{LAP}}$, only $u_{0}$ is variable. Considering that the right-hand side is convex in $u_{0}$, we can find $\bar{u}_{0}$ which satisfies Constraint (10. a) by applying Newton's method in $u_{0}$.

2. Finding feasible $v_{1}^{j}(j \in J)$ via Constraint (10.d). By substituting $\boldsymbol{w}^{\mathrm{LAP}}$ and $\bar{u}_{0}$ in Constraint (10.d), $\bar{v}_{1}^{j}(j \in J)$ are determined.

3. Finding feasible $u_{1}^{j}(j \in J)$ via Constraint (10.b). Now in Constraint (10. b), only $u_{1}^{j}(j \in J)$ are variables. In the same manner as in case of Constraint (10. a), we can find $u_{1}^{j}(j \in J)$ by applying Newton's method $m=|J|$ times.

4. Finding feasible $v_{\tau}^{j}(\tau \in T \backslash\{1\}, j \in J)$ and $u_{\tau}^{j}(\tau \in T \backslash\{1, t\}, j \in J)$ via Constraints (10.e) and (10. c). In the same way as described above, by repeating the following procedures until $\tau=t$ is satisfied, we obtain a feasible solution $\left(\boldsymbol{w}^{\mathrm{LAP}}, \overline{\boldsymbol{u}}, \overline{\boldsymbol{v}}\right)$ to Problem (12).

- By substituting $\boldsymbol{w}^{\mathrm{LAP}}$ and $\bar{u}_{\tau-1}^{j}(j \in J)$ in Constraint $(10 . \mathrm{e}), \bar{v}_{\tau}^{j}(j \in J)$ are determined.

- In Constraint (10. c), if we substitute $\boldsymbol{w}^{\mathrm{LAP}}, \bar{u}_{\tau-1}^{j}(j \in J)$ and $\bar{v}_{\tau}^{j}(j \in J)$, we can find $\bar{u}_{\tau}^{j}(j \in J)$ by applying Newton's method $m$ times.

5. Evaluation of objective value. Employing the obtained $\bar{v}_{t}^{j}(j \in J)$ in the above procedures, the objective value of Problem (12) is calculated.

\subsection{Outline of Algorithmic Steps}

We are now in a position to describe the local search algorithm. 
Local Search Algorithm for Problem (12)

Step 0. [Initialization.] Set $\overline{\boldsymbol{w}}$ which is feasible to Problem (12) and the maximum number of iterations.

Step 1. [Newton's method.] Substitute $\overline{\boldsymbol{w}}$ in Equations (10), and find a feasible solution to Problem (12) by solving Equations (10) via Newton's method. Set the obtained solution as $(\overline{\boldsymbol{u}}, \overline{\boldsymbol{v}})$.

Step 2. [ Termination check. ] If the evaluated objective value is not improved or the maximum number of iterations is reached, terminate the algorithm with the best solution obtained so far. Otherwise, go to Step 3.

Step 3. [Linear approximation problem.] Solve the linear approximation problem $\operatorname{LAP}(\overline{\boldsymbol{u}}, \overline{\boldsymbol{w}})$, and let $\left(\boldsymbol{u}^{\mathrm{LAP}}, \boldsymbol{v}^{\mathrm{LAP}}, \rho^{\mathrm{LAP}}, \boldsymbol{w}^{\mathrm{LAP}}, \boldsymbol{z}^{\mathrm{LAP}}\right)$ be a solution to it. Set $\overline{\boldsymbol{w}} \leftarrow$ $\boldsymbol{w}^{\mathrm{LAP}}$, and go to Step 1.

\section{Computational Results}

In this section, we show computational results and evaluate the effectiveness of the proposed algorithm and the performance of the constant rebalancing strategy. All computations are conducted on a Windows XP personal computer with AMD Athlon 64 Processor $(2.41 \mathrm{GHz})$ and 2GB memory, and NUOPT (ver.10.1.4), a mathematical programming software package developed by Mathematical System, Inc., is employed for solving all the optimization problems.

Problem Setting. Ten financial assets are considered over five periods, and the number of scenarios (simulated paths) is 1,000 (i.e., $n:=10, t:=5$ and $m:=1,000$ ). Asset $i=1$ is cash with no transaction cost, and Assets $i=2,3,4,8,10$ are low-risk assets with low transaction costs (e.g., bond), and Assets $i=5,6,7,9$ are high-risk assets with high transaction costs (e.g., stock) as shown in Figure 5. On the advice of Mizuho-DL Financial Technology Co., Ltd., the parameters regarding transaction costs are estimated using historical data, and the value of price and dividend in each scenario are generated via a bootstrap method. Lower limits of investment proportion are all zero (i.e., $\boldsymbol{w}^{L}:=\mathbf{0}$ ), and the initial investment unit $\overline{\boldsymbol{y}}$ is set as $\overline{\boldsymbol{y}}:=\mathbf{0}$, and the initial wealth $v_{0}$ is 1.0 trillion Japanese yen.

Parameter Setting of the Local Search Algorithm. Step size parameters $\kappa_{i}$ are set as $\kappa_{1}:=1, \kappa_{i}:=0.1(i \in I \backslash\{1\})$, and let the maximum number of iterations be eleven, i.e., the linear approximation problem is solved ten times so that all investment proportions can be reached from any starting value, $\overline{\boldsymbol{w}}$, with step size of 0.1 . 


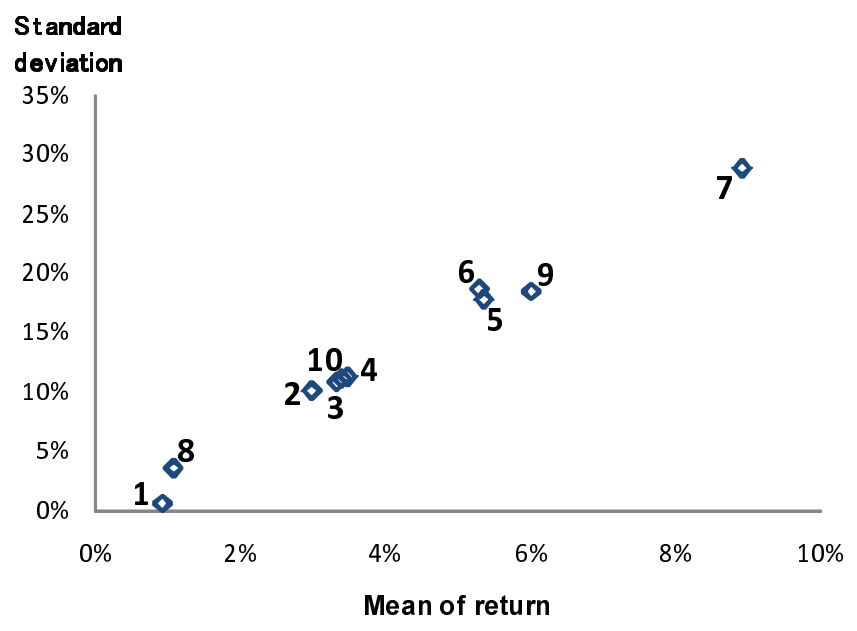

Figure 5: Mean and Standard Deviation of Asset Return

\subsection{Comparison with the Rectangular Branch-and-Bound Algorithm}

Maranas et al. (1997) proposed a rectangular branch-and-bound algorithm for globally solving the constant rebalanced mean-variance portfolio optimization. In this subsection, we revise their algorithm so that mean-CVaR portfolio optimization in this paper can be solved, and compare the performance of the proposed local search algorithm with that of the revised version of the branch-and-bound algorithm.

\subsubsection{Rectangular Branch-and-Bound Algorithm in Maranas et al. (1997)}

When no transaction cost is considered, one has $\boldsymbol{u}=\boldsymbol{v}$ in Constraints (10.a), (10.b) and (10.c), and accordingly, decision variable $\boldsymbol{u}$ can be eliminated. Then, by eliminating $\boldsymbol{v}$, the multiplicative return can be explicitly represented in the objectives, and Problem (12) is reduced to the following formulation with only $n+1$ variables and simple linear constraints:

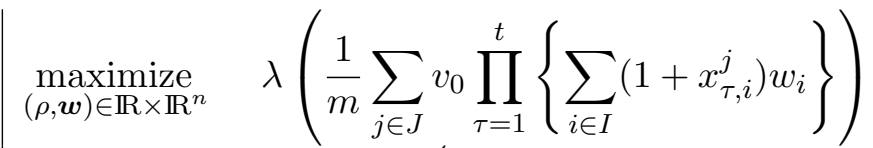

$$
\begin{aligned}
& -(1-\lambda)\left(\rho+\frac{1}{(1-\beta) m} \sum_{j \in J} \phi\left(-v_{0} \prod_{\tau=1}^{t}\left\{\sum_{i \in I}\left(1+x_{\tau, i}^{j}\right) w_{i}\right\}-\rho\right)\right)
\end{aligned}
$$

subject to Investment Proportion Constraint (11), 


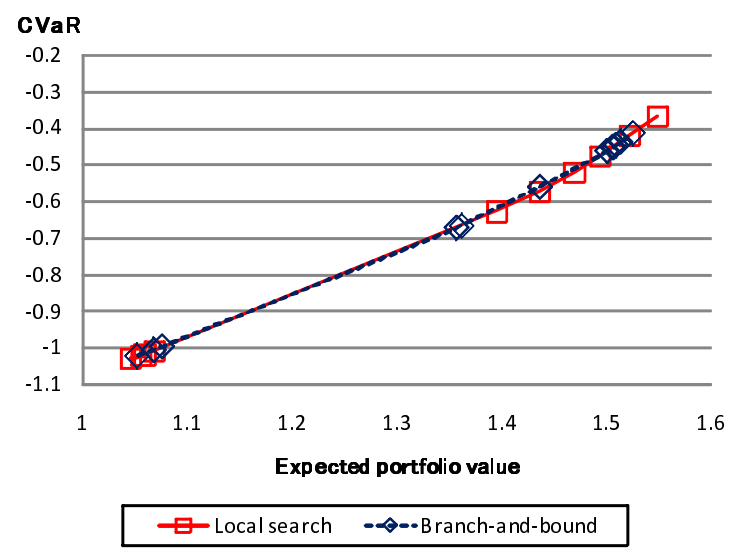

(a) Under no transaction costs

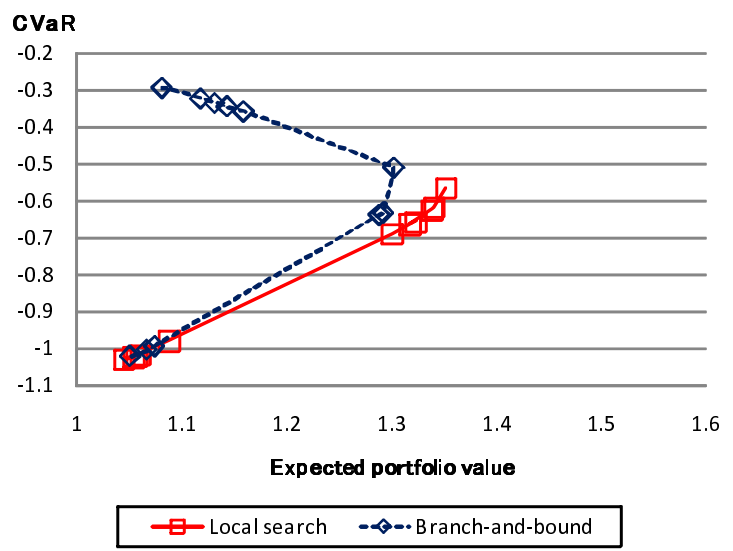

(b) Under transaction costs

Figure 6: Efficient Frontier by Applying the Local Search Algorithm and the Rectangular Branch-and-Bound Algorithm

where $\phi: \mathbb{R} \rightarrow \mathbb{R}$ is a smoothing function of nondifferentiable plus function $[\xi]^{+}$. In this paper, we adopt

$$
\phi(\xi):=\frac{\sqrt{\xi^{2}+4 \delta^{2}}+\xi}{2},
$$

where $\delta>0$ is a parameter representing approximation accuracy (Pang and Leyffer, 2004). A rectangular branch-and-bound algorithm proposed in Maranas et al. (1997) is a solution method for the constant rebalanced mean-variance portfolio optimization under no transaction costs, where the feasible region is partitioned into rectangles. The algorithm is explained in Maranas et al. (1997) in detail, and a revised version of convex subproblem over the subrectangle is shown in Appendix B. When the number of assets, $n$, is small, Problem (16) is a small size problem and the algorithm works well. However, Problem (16) is not easily solved when the number of assets, $n$, is large, and in addition, variable elimination mentioned above is impossible under transaction costs.

\subsubsection{Discussion on Results}

Efficient Frontier. Figure 6 shows the efficient frontier of the solutions obtained by the two algorithms. The horizontal axis is the expected portfolio value at the end of period $t$, that is $\frac{1}{m} \sum_{j \in J} v_{t}^{j}$, and the vertical axis is the CVaR representing a risk of decrease in portfolio value at the end of period $t$, that is $\min \left\{\rho+\frac{1}{(1-\beta) m} \sum_{j \in J}\left[-v_{t}^{j}-\rho\right]^{+} \mid \rho \in \mathbb{R}\right\}$. We choose $\lambda$ from $\{0.01,0.5,0.525,0.55,0.575,0.6,0.625,0.65,0.675,0.7,0.99\}$. 


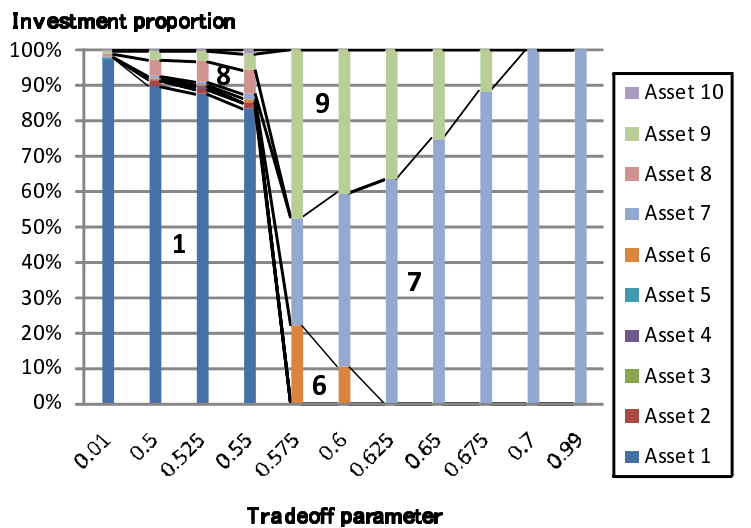

(a) Under no transaction costs

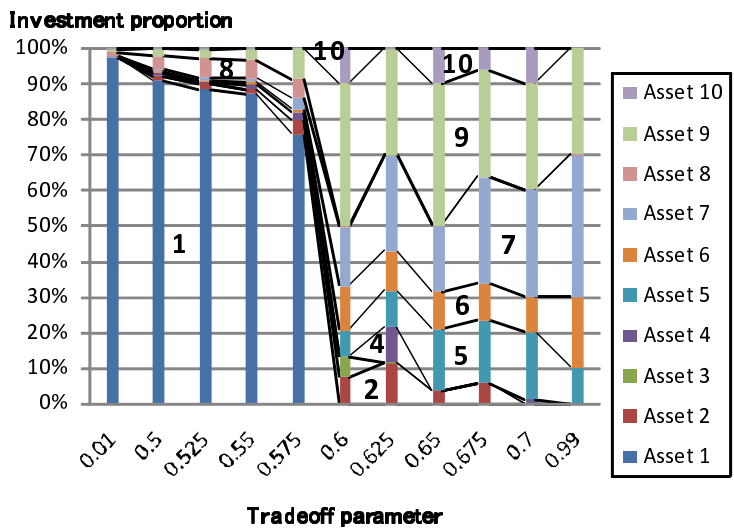

(b) Under transaction costs

Figure 7: Optimal Investment Proportion Provided by the Local Search Algorithm

Figure 6 (a) depicts two efficient frontiers under no transaction costs. We see that the two frontiers almost coincide, which indicates that the local search algorithm attains almost optimal solutions in the sense that the branch-and-bound algorithm achieves the global optimality ${ }^{1}$. Next, Figure 6 (b) shows the results under transaction costs, where the efficient frontier of the branch-and-bound algorithm is drawn with the solutions to Problem (16) in which no transaction cost is considered. We see that the branch-and-bound algorithm can provide highly inefficient solutions, and especially if one seeks high return, large transaction costs occur through the investment. This result implies that solving the problem under no transaction costs results in an insufficient investment in the presence of transaction costs. Consequently, the problem needs to be solved by applying the local search algorithm if transaction costs occur through the investment.

Optimal Investment Proportion. Figure 7 shows the optimal investment proportion provided by the local search algorithm. Comparing the results under no transaction costs (Figure 7 (a)) with those under transaction costs (Figure 7 (b)), they are similar in that investment proportion in Asset 1 (cash) is large in case of small $\lambda$ (i.e., low-risk investment). On the other hand, in case of large $\lambda$ (i.e., high-return investment), investment proportion in Asset 7 is large under no transaction costs, and investments are diversified among four or five assets under transaction costs. If investment proportion in an asset is so large, large transaction costs are incurred, and hence by diversification, transaction costs are small. 


\subsection{Comparison with the Buy-and-Hold Strategy}

Buy-and-hold strategy is an investment strategy in which investor buys financial assets at the beginning and holds them until the end of planning period without trading. This strategy is popular and simple, and therefore, can be a benchmark. In this subsection, we compare the performance of the two strategies, both of which take the transaction costs into account.

\subsubsection{Buy-and-Hold Strategy}

Portfolio optimization problem with buy-and-hold strategy under transaction costs is formulated as the following problem:

$$
\begin{aligned}
& \underset{\boldsymbol{\rho}, \boldsymbol{v}, \boldsymbol{y}}{\operatorname{maximize}} \frac{1}{m} \sum_{j \in J} v_{t}^{j} \\
& \text { subject to } \rho_{1}+\frac{1}{(1-\beta) m} \sum_{j \in J}\left[v_{0}-v_{1}^{j}-\rho_{1}\right]^{+} \leq \alpha_{1} \text {, } \\
& \rho_{\tau}+\frac{1}{(1-\beta) m} \sum_{j \in J}\left[v_{\tau-1}^{j}-v_{\tau}^{j}-\rho_{\tau}\right]^{+} \leq \alpha_{\tau}, \quad \tau \in T \backslash\{1\} \\
& \sum_{i \in I} p_{0, i} y_{i}+\sum_{i \in I} \gamma_{i}\left(p_{0, i} y_{i}-p_{0, i} \bar{y}_{i}\right) \leq v_{0} \\
& v_{\tau}^{j}=\sum_{i \in I}\left(p_{\tau, i}^{j} y_{i}+\sum_{\theta=1}^{\tau} d_{\theta, i}^{j} y_{i}\right), \quad \tau \in T, j \in J \\
& y_{i}^{L} \leq y_{i} \leq y_{i}^{U}, \quad i \in I,
\end{aligned}
$$

where $y_{i}$ denotes investment unit in asset $i \in I$, and $y_{i}^{L}\left(y_{i}^{U}\right)$ is a lower (upper) limit of investment unit in asset $i \in I$. In the paper, lower limits of investment unit are all zero (i.e., $\boldsymbol{y}^{L}:=\mathbf{0}$ ). Since the transaction cost is represented by the convex function (1), Problem (18) is a convex problem and is solved by employing NUOPT, an NLP solver mentioned above. In this problem, the decision variables for representing portfolio are not investment proportion $\boldsymbol{w}$, but investment unit $\boldsymbol{y}$, and portfolio value $v_{\tau}^{j}(\tau \in T, j \in J)$ is defined as the sum of asset values in market at the end of period $\tau$ and dividends obtained by the end of period $\tau$.

\subsubsection{Discussion on Results}

Efficient Frontier. Figure 8 shows the efficient frontier of the two strategies. Upper bounds of $\mathrm{CVaR}, \alpha_{\tau}$, at periods $\tau \in T$ are all the same (i.e., $\alpha_{\tau}:=\alpha, \tau \in T$ ), and we choose $\alpha$ from $\{0.05,0.1,0.2,0.3,0.4,0.5,0.6,1\}$ in case of no transaction costs and from $\{0.05,0.1,0.15$, 


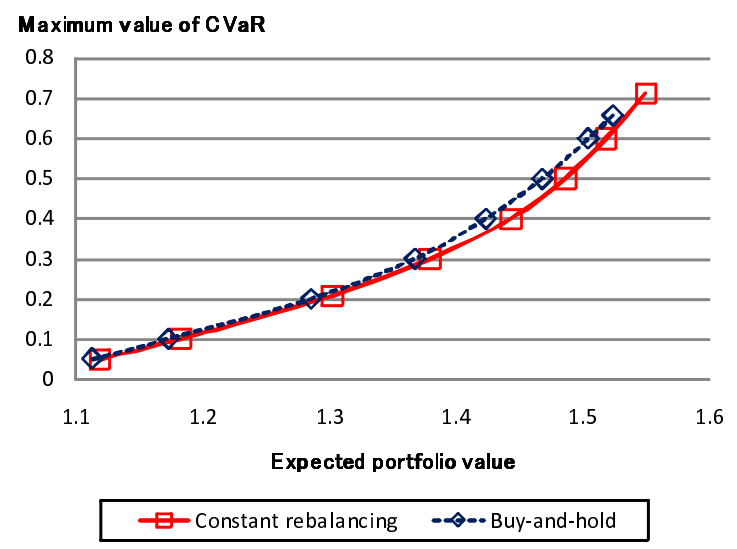

(a) Under no transaction costs

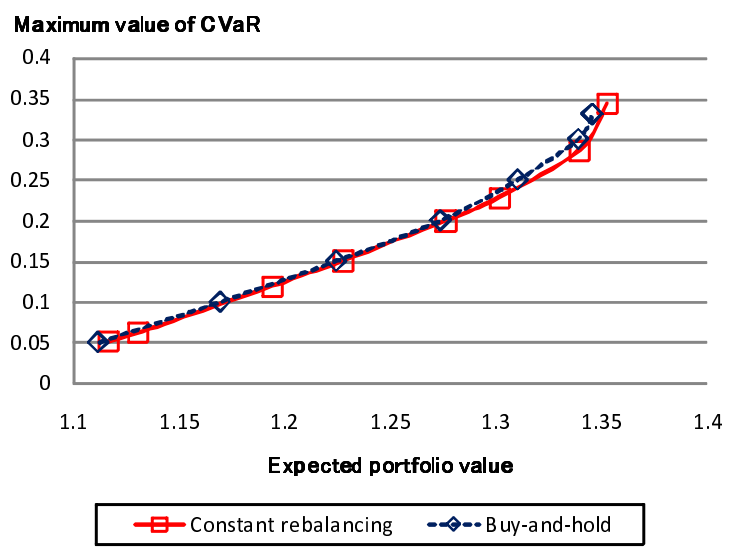

(b) Under transaction costs

Figure 8: Efficient Frontier of the Constant Rebalancing Strategy and the Buy-and-Hold Strategy

$0.2,0.25,0.3,0.35,1\}$ in case of transaction costs. "Constant rebalancing" and "Buy-and-hold" correspond to the solutions of Problem (13) via our local search algorithm ${ }^{2}$ and Problem (18) via the NLP solver, respectively. Then, the horizontal axis is the expected portfolio value at the end of period $t$, and the vertical axis is the maximum value of $\mathrm{CVaR}$ with respect to period $\tau \in T$.

Comparing the results under no transaction costs (Figure 8 (a)), the two frontiers are almost the same at the left part of the figure (i.e., low-risk investment). On the other hand at the right part of the figure (i.e., high-return investment), the constant rebalancing strategy dominates the buy-and-hold strategy. One reason for this is that the obtained dividends can be invested in high-return assets in case of constant rebalancing. Next, let us consider the results under transaction costs (Figure 8 (b)). The frontier provided by the constant rebalancing strategy dominates that provided by the buy-and-hold strategy at the right part of the figure (i.e., highreturn investment); however, the difference is small compared to the results under no transaction costs. It follows from that since rebalancing requires transaction costs, the performance of the constant rebalancing deteriorates somewhat.

Optimal Investment Proportion. Figure 9 shows the optimal investment proportion of the constant rebalancing strategy and the buy-and-hold strategy. The two strategies provide similar investment proportion under no transaction costs (Figure 9 (a) and (c)) and under transaction 


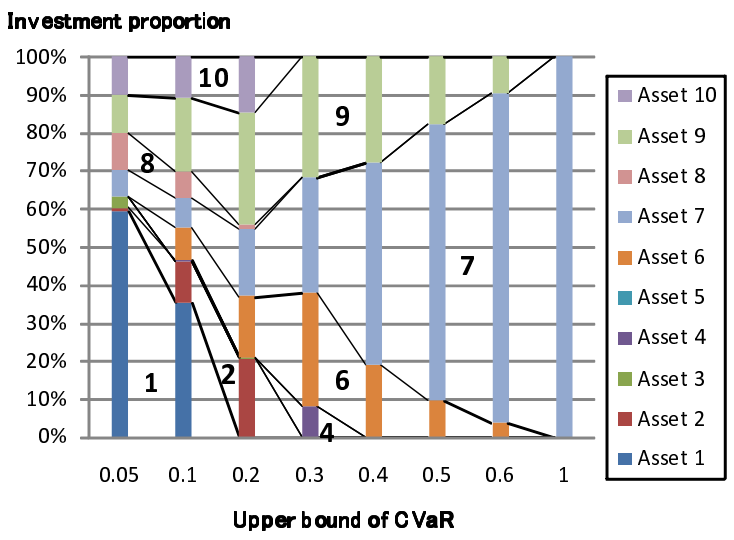

(a) Constant rebalancing (under no transaction costs)

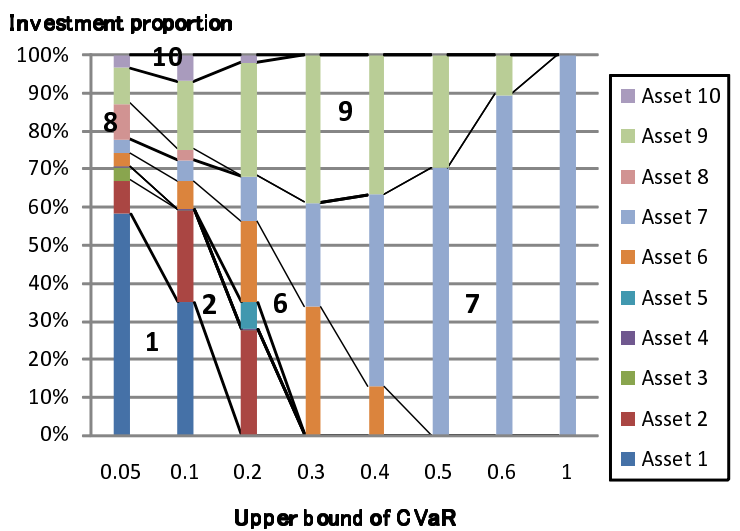

(c) Buy-and-hold (under no transaction costs)

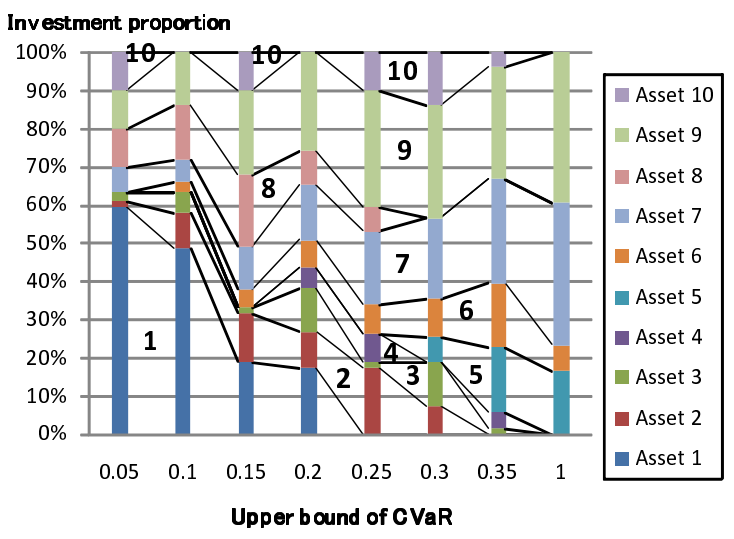

(b) Constant rebalancing (under transaction costs)

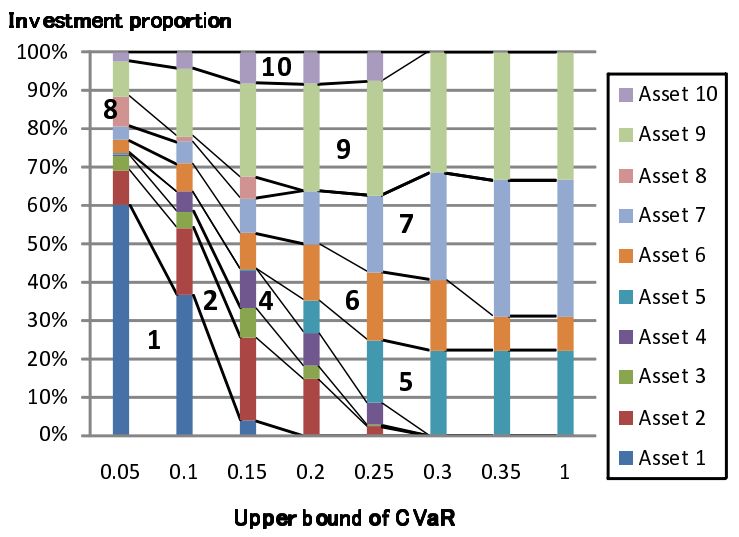

(d) Buy-and-hold (under transaction costs)

Figure 9: Optimal Investment Proportion of the Constant Rebalancing Strategy and the Buyand-Hold Strategy

costs (Figure 9 (b) and (d)), respectively. The smaller the upper bound of CVaR is, the larger the investment proportion in cash becomes, and the larger the upper bound of CVaR is, the larger the investment proportions in high-risk assets become. In addition, whereas investment proportion in Asset 7 is so large under no transaction costs, investments are diversified among four to six assets under transaction costs.

Computational Time. In drawing the efficient frontier in Figure 8 (a) or (b), eight problems are solved with different parameter values. For drawing the efficient frontier of the constant rebalancing strategy, we sequentially solve the problems by gradually increasing the parameter 


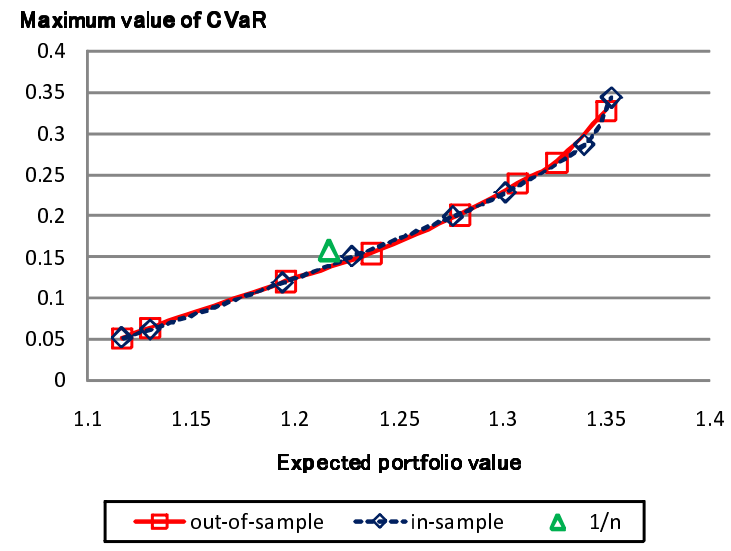

(a) Results of scenario set A

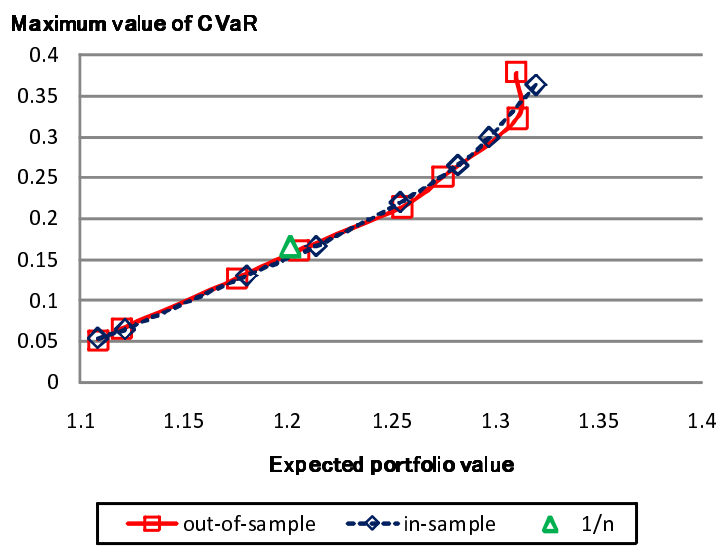

(b) Results of scenario set B

[ out-of-sample] an optimal investment proportion of Problem (13) using the scenario set which is different from what we use for evaluating the performance; [in-sample] an optimal investment proportions of Problem (13) using the same scenario set with what we use for evaluating the performance; $[1 / n]$ an equally weighted portfolio, that is $\boldsymbol{w}=(1 / n, 1 / n, \ldots, 1 / n)$.

Figure 10: Out-of-Sample Performance of the Constant Rebalancing Strategy under Transaction Costs

of the upper bound of $\mathrm{CVaR}, \alpha$. The obtained solution under the previous $\alpha$ is employed as the initial solution to the problem for the next $\alpha$. First, starting value of investment proportion, $\overline{\boldsymbol{w}}$, is set as $\bar{w}_{1}:=1, \bar{w}_{i}:=0(i \in I \backslash\{1\})$, and the lowest risk investment problem (say, $\alpha=0.05$ ) is solved. This investment proportion $\overline{\boldsymbol{w}}$ satisfies Constraint (11) and probably CVaR constraints of Problem (13) since cash is the lowest risk asset. Next, the obtained solution is employed as a starting value, $\overline{\boldsymbol{w}}$, and the second lowest risk investment problem (say, $\alpha=0.1$ ) is solved. Repeating this procedure to the highest return investment problem (say, $\alpha=1$ ), an efficient frontier is drawn with the small number of iterations of the algorithm. In the experiments, the algorithm for constant rebalancing terminates with three iterations, i.e., the linear approximation problem is solved only twice for all the problems under transaction costs; the average CPU time is 287.6 seconds, whereas the average CPU time of the buy-and-hold strategy is 18.5 seconds.

\subsection{Out-of-Sample Performance}

In this subsection, we conduct experiments for evaluating the out-of-sample performance of the constant rebalancing strategy under transaction costs. Scenario sets A and B, each containing 1,000 scenarios, are generated via a bootstrap method using the same historical data. Figure 
10 shows the efficient frontiers where the setting is the same as that in Figure 8 (b). In the results using the scenario set B (Figure 10 (b)), the frontier of out-of-sample solutions differs from that of in-sample solutions at high-return points, however the two frontiers are almost the same. Then, although it has been shown in recent papers (e.g., DeMiguel et al., 2009) that equally weighted portfolio performs well in out-of-sample tests, the performance of $1 / n$-portfolio is dominated by that of the optimization problem. This is common in the observation reported in Fleten et al. (2002) that the constant rebalancing approach performs better in the out-of-sample result than in the in-sample result compared to the stochastic dynamic approach.

\section{Conclusion}

In this paper, we formulate the constant rebalanced portfolio optimization problem under nonlinear transaction costs, and propose a solution method based on a local search approach. This problem is a nonconvex optimization including a large number of bilinear terms of decision variables in a number of constraints and difficult to attain a globally optimal solution in general. When a huge number of scenarios are considered, it becomes further difficult to attain a locally optimal solution via a state-of-the-art NLP solver. Then, we propose an iterative local search algorithm based on LP solution, which is easily attained even if the problem size is large, and on Newton's method for solving nonlinear equations. In the computational results, the proposed local search algorithm attains as good solution as the global optimization approach. Then, we show that the problem under transaction costs needs to be solved so as to obtain an efficient solution. Moreover, we see that the constant rebalancing strategy outperforms the buy-and-hold strategy when high return is sought. Furthermore in the out-of-sample performance, the constant rebalancing strategy is superior to the equally weighted portfolio. In addition, it should be noted that the proposed local search algorithm can deal with general nonlinear transaction costs.

Future tasks include improving the efficiency of the solution method and comparing the out-of-sample performance of the constant rebalancing strategy with that of the other various strategies. In a practical situation, the problem with a large number of scenarios (e.g., 10,000 or 100,000 scenarios) is desired to be solved. Therefore, we need to improve the algorithm for solving the problem with a large number of scenarios. On the other hand, dynamic stochastic approach (e.g. Infanger, 2006), hybrid model (Hibiki, 2003, 2006) and the like are not tested for comparing 
with the constant rebalancing strategy in this paper. Although these are partly conducted in (Fleten et al., 2002; Hibiki, 2006), more detailed and inclusive comparison is essential for showing the effectiveness of the constant rebalancing strategy especially in out-of-sample performance.

\section{Acknowledgments}

We wish to thank Mizuho-DL Financial Technology Co., Ltd., for generous financial assistance, and Professor Yoshitsugu Yamamoto from University of Tsukuba for helpful suggestions and comments. This work was supported by Grant-in-Aid for JSPS Fellows. Research of the second author is partly supported by MEXT Grant-in-Aid for Young Scientists (B) 20710120, and by Chuo University Grant for Special Research. 


\section{Appendix A}

Linearly Approximated Portfolio Dynamics Equation. In order to formulate $\operatorname{LAP}(\overline{\boldsymbol{u}}, \overline{\boldsymbol{w}})$ which is a linear approximation problem for Problem (12), the nonlinear terms of decision variables in Constraint (10) are approximated in a linear manner at $(\overline{\boldsymbol{u}}, \overline{\boldsymbol{w}})$ with respect to $\boldsymbol{u}$, $\boldsymbol{w}$ as follows:

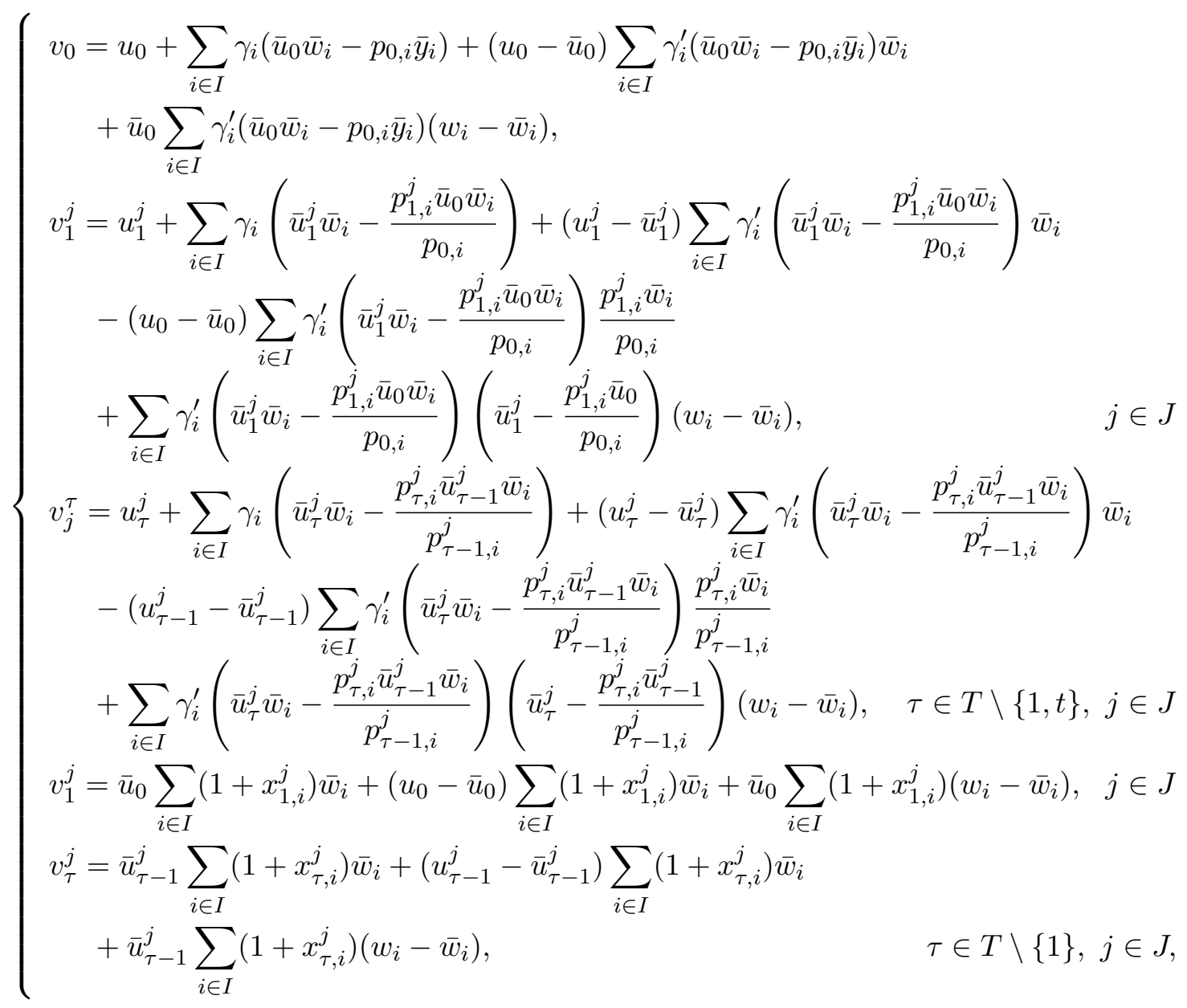

where $\gamma_{i}^{\prime}: \mathbb{R} \rightarrow \mathbb{R}(i \in I)$ represents a subderivative of $\gamma_{i}$, that is

$$
\gamma_{i}^{\prime}(\xi):= \begin{cases}\left(d_{i} \xi+1\right) c_{i} \exp \left(d_{i} \xi\right) & \text { if } \xi>0 \\ 0 & \text { if } \xi=0 \\ \left(b_{i} \xi-1\right) a_{i} \exp \left(-b_{i} \xi\right) & \text { if } \xi<0\end{cases}
$$




\section{Appendix B}

Convex Subproblem over the Subrectangle. In the rectangular branch-and-bound algorithm for Problem (16), the problem over a subrectangle $\left[\boldsymbol{w}^{\mathcal{L}}, \boldsymbol{w}^{\mathcal{U}}\right]$ is relaxed by adding the extra quadratic term to the objective function:

$$
\begin{aligned}
\underset{(\rho, \boldsymbol{w}) \in \mathbb{R} \times \mathbb{R}^{n}}{\operatorname{maximize}} & \lambda\left(\frac{1}{m} \sum_{j \in J} v_{0} \prod_{\tau=1}^{t}\left\{\sum_{i \in I}\left(1+x_{\tau, i}^{j}\right) w_{i}\right\}\right) \\
& -(1-\lambda)\left(\rho+\frac{1}{(1-\beta) m} \sum_{j \in J} \phi\left(-v_{0} \prod_{\tau=1}^{t}\left\{\sum_{i \in I}\left(1+x_{\tau, i}^{j}\right) w_{i}\right\}-\rho\right)\right) \\
& -\alpha \sum_{i \in I}\left(w_{i}^{\mathcal{L}}-w_{i}\right)\left(w_{i}^{\mathcal{U}}-w_{i}\right)
\end{aligned}
$$

subject to Investment Proportion Constraint (11),

$$
w_{i}^{\mathcal{L}} \leq w_{i} \leq w_{i}^{\mathcal{U}}, \quad i \in I,
$$

where $\alpha>0$ is a scalar parameter.

Proposition 1 Function (21. a) is concave in $(\rho, \boldsymbol{w})$ for a sufficiently large $\alpha$.

Proof. Let $\boldsymbol{H}=\left(h_{i j}\right) \in \mathbb{R}^{(1+n) \times(1+n)}$ be a Hessian matrix of " $(-1) \times($ Function (21. a))" with respect to $(\rho, \boldsymbol{w})$. To complete the proof it is only necessary to show that $\boldsymbol{H}$ is positive semidefinite. Because $\phi^{\prime \prime}(\cdot)>0$ due to a property of smoothing function (Pang and Leyffer, 2004), we have

$$
\begin{aligned}
h_{11} & =\frac{\partial^{2}}{\partial \rho \partial \rho}((-1) \times(\text { Function }(21 . \mathrm{a}))) \\
& =\frac{(1-\lambda)}{(1-\beta) m} \sum_{j \in J} \phi^{\prime \prime}\left(-v_{0} \prod_{\tau=1}^{t}\left\{\sum_{i \in I}\left(1+x_{\tau, i}^{j}\right) w_{i}\right\}-\rho\right) \geq 0 .
\end{aligned}
$$

We show that $(\rho, \boldsymbol{w})^{\top} \boldsymbol{H}(\rho, \boldsymbol{w}) \geq 0$ for all $(\rho, \boldsymbol{w})$. If $\boldsymbol{w} \neq \mathbf{0}$,

$$
(\rho, \boldsymbol{w})^{\top} \boldsymbol{H}(\rho, \boldsymbol{w})=2 \alpha \sum_{i=1}^{n} w_{i}^{2}+\underbrace{\ldots \ldots}_{\alpha \text { does not appear }},
$$

is nonnegative for a sufficiently large $\alpha$. Otherwise $\boldsymbol{w}=\mathbf{0}$, and then we have $(\rho, \boldsymbol{w})^{\top} \boldsymbol{H}(\rho, \boldsymbol{w})=$ $\rho^{2} h_{11} \geq 0$. 


\section{Notes}

\footnotetext{
${ }^{1}$ In Appendix B, the subproblems are proved to be convex when a parameter $\alpha$ is sufficiently large. It is, however, difficult to ascertain whether the parameter value is properly set (see Maranas and Floudas, 1994, for the details) and accordingly, it is possible that a globally optimal solution may not be reached in experimental results.

${ }^{2}$ Although the proposed algorithm does not necessarily attain a solution satisfying the CVaR constraints, the attained solution almost satisfies them.
}

\section{References}

Bertsimas, D. \& Pachamanova, D. (2008). Robust Multiperiod Portfolio Management in the Presence of Transaction Costs. Computers \& Operations Research, 35, 3-17.

Brandt, M.W. (1999). Estimating Portfolio and Consumption Choice: A Conditional Euler Equations Approach. Journal of Finance, 54, 1609-1645.

Brennan, M.J., Schwartz, E.S. \& Lagnado, R. (1997). Strategic Asset Allocation. Journal of Economic Dynamics \& Control, 21, 1377-1403.

Calafiore, G.C. (2008). Multi-Period Portfolio Optimization with Linear Control Policies. Automatica, 44, 2463-2473.

Cariño, D.R., Kent, T., Myers, D.H., Stacy, C., Sylvanus, M., Turner, A.L., Watanabe, K. \& Ziemba, W.T. (1994). The Russell-Yasuda Kasai Model: An Asset/Liability Model for a Japanese Insurance Company Using Multistage Stochastic Programming. Interfaces, 24, $29-49$.

Cornuejols, G. \& Tütüncü, R. (2007). Optimization Methods in Finance. (Cambridge University Press)

Cover, T.M. (1991). Universal Portfolios. Mathematical Finance, 1, 1-29.

Dantzig, G.B. \& Infanger, G. (1993). Multi-Stage Stochastic Linear Programs for Portfolio Optimization. Annals of Operations Research, 45, 59-76.

DeMiguel, V., Garlappi, L. \& Uppal, R. (2009). Optimal Versus Naive Diversification: How Inefficient is the $1 / N$ Portfolio Strategy? Review of Financial Studies, 22, 1915-1953.

Ermoliev, Y. \& Wets, R.J.-B. (Eds.) (1988). Numerical Techniques for Stochastic Optimization. (Springer-Verlag.) 
Fleten, S.-E., Høyland, K. \& Wallace, S.W. (2002). The Performance of Stochastic Dynamic and Fixed Mix Portfolio Models. European Journal of Operational Research, 140, 37-49.

Gaivoronski, A.A. \& Stella, F. (2003). On-Line Portfolio Selection Using Stochastic Programming. Journal of Economic Dynamics \& Control, 27, 1013-1043.

Hibiki, N. (2003). A Hybrid Simulation/Tree Stochastic Optimisation Model for Dynamic Asset Allocation. (In B. Scherer (Eds.), Asset and Liability Management Tools: A Handbook for Best Practice (pp.269-294). Risk Books.)

Hibiki, N. (2006). Multi-Period Stochastic Optimization Models for Dynamic Asset Allocation. Journal of Banking \&3 Finance, 30, 365-390.

Infanger, G. (2006). Dynamic Asset Allocation Strategies Using a Stochastic Dynamic Programming Approach. (In S.A. Zenios \& W.T. Ziemba (Eds.), Handbook of Asset and Liability Management, (pp.199-251). Elsevier.)

Konno, H., Thach, P.T. \& Tuy, H. (1997). Optimization on Low Rank Nonconvex Structures. (Dordrecht: Kluwer Academic)

Kusy, M.I. \& Ziemba, W.T. (1986). A Bank Asset and Liability Management Model. Operations Research, 34, 356-376.

Maranas, C.D. \& Floudas, C.A. (1994). Global Minimum Potential Energy Conformations of Small Molecules. Journal of Global Optimization, 4, 135-170.

Maranas, C.D., Androulakis, I.P., Floudas, C.A., Berger, A.J. \& Mulvey, J.M. (1997). Solving Long-Term Financial Planning Problems via Global Optimization. Journal of Economic Dynamics \& Control, 21, 1405-1425.

Markowitz, H. (1952). Portfolio Selection. Journal of Finance, 7, 77-91.

Markowitz, H. (1991). Portfolio Selection: Efficient Diversification of Investments (2nd edition). (New York: Wiley.)

Merton, R.C. (1969). Lifetime Portfolio Selection under Uncertainty: The Continuous-Time Case. Review of Economics and Statistics, 51, 247-257. 
Merton, R.C. (1971). Optimum Consumption and Portfolio Rules in a Continuous-Time Model. Journal of Economic Theory, 3, 373-413.

Mulvey, J.M. \& Vladimirou, H. (1989). Stochastic Network Optimization Models for Investment Planning. Annals of Operations Research, 20, 187-217.

Mulvey, J.M. \& Ziemba, W.T. (1995). Asset and Liability Allocation in a Global Environment. (In R.A. Jarrow, V. Maksimovic \& W.T. Ziemba (Eds.), Finance (Handbooks in Operations Research and Management Science, Vol. 9), (pp.435-463). North-Holland.)

Pang, J.-S. \& Leyffer, S. (2004). On the Global Minimization of the Value-at-Risk. Optimization Methods and Software, 19, 611-631.

Perold, A.F. \& Sharpe, W.F. (1995). Dynamic Strategies for Asset Allocation. Financial Analysts Journal, 51, 149-160.

Pflug, G.Ch. (2000). Some Remarks on the Value-at-Risk and the Conditional Value-at-Risk. (In S. Uryasev (Eds.), Probabilistic Constrained Optimization: Methodology and Applications, (pp.272-281). Dordrecht: Kluwer Academic Publishers.)

Pinar, M.Ç. (2007). Robust Scenario Optimization Based on Downside-Risk Measure for MultiPeriod Portfolio Selection. OR Spectrum, 29, 295-309.

Rockafellar, R.T. \& Uryasev, S. (2002). Conditional Value-at-Risk for General Loss Distributions. Journal of Banking \& Finance, 26, 1443-1471.

Samuelson, P.A. (1969). Lifetime Portfolio Selection by Dynamic Stochastic Programming. Review of Economics and Statistics, 51, 239-246.

Skaf, J. \& Boyd, S. (2008). Multi-Period Portfolio Optimization with Constraints and Transaction Costs. Working Paper, Stanford University.

Tuy, H. (1998). Convex Analysis and Global Optimization. (Dordrecht, Kluwer Academic Publishers)

Zhang, X.-L. \& Zhang, K.-C.(2009). Using Genetic Algorithm to Solve a New Multi-Period Stochastic Optimization Model. Journal of Computational and Applied Mathematics, 231, $114-123$. 
Ziemba, W.T. \& Mulvey, J.M. (Eds.) (1998). Worldwide Asset and Liability Modeling. (Cambridge University Press) 